\title{
The plane mixed problem for an elastic semi-strip under different load types at its short edge
}

\author{
O. Menshykov · O. Reut · V. Reut · \\ N. Vaysfeld · Z. Zhuravlova
}

Received: date / Accepted: date

\begin{abstract}
The mixed problem for the fixed semi-strip is investigated in this article for the three cases of the applied mechanical load. The solution of the boundary problem is reduced to the solution of the singular integral equation (SIE) with regard to the unknown displacements derivative. Three cases of SIE are investigated: when the mechanical load is applied on the center of the semi-strips edge, when the mechanical load is distributed near the left lateral side and when the mechanical load is distributed on the whole semi-strip's edge. In the first case SIE is solved by the using of the orthogonal polynomials method. In the second and third cases the characteristical equations to SIE are constructed, and the SIE are solved with the help of the generalized method. The stress state of the semi-strip is investigated for the three cases.
\end{abstract}

Keywords semi-strip · singular integral equation · fixed singularity · orthogonal polynomials method $\cdot$ generalized method

O. Menshykov

University of Aberdeen

E-mail: o.menshykov@abdn.ac.uk

V. Reut

Odessa Mechnikov University, Institute of Mathematics, Economics and Mechanics, str. Dvoryanskaya, 2, 65082, Odessa, Ukraine

E-mail: reut@onu.edu.ua

N. Vaysfeld

Odessa Mechnikov University, Institute of Mathematics, Economics and Mechanics, str. Dvoryanskaya, 2, 65082, Odessa, Ukraine

Tel.: +38-050-316-12-70

Fax: $+38-048-723-3957$

E-mail: vaysfeld@onu.edu.ua

Z. Zhuravlova

Odessa Mechnikov University, Institute of Mathematics, Economics and Mechanics, str. Dvoryanskaya, 2, 65082, Odessa, Ukraine

Tel.: +38-067-518-96-20

E-mail: z.zhuravlova@onu.edu.ua 


\section{Introduction}

The plane elasticity problems for a semi-strip are useful for many engineering application. It explains the big amount of works dedicated to these problems.

The method of block element was used in [1]. The superposition method of semi-plane and infinite strip was applied in [2] to achieve solution for the semi-strip. The approach based on the construction of the stress function as the combination of the Fouriers integrals and the series was used in [3], [4], [5]. The reducing of the problem to the Fredholm's integral equation of the first kind was applied in [6]. The problems for a semi-strip in [7], [8], [9] were reduced to the SIE. It was solved numerically by reduction to the solving of an infinity system of the algebraic equations. The method based on diagonalization of the equilibrium equations system was proposed in [10]. The variation method was used in [11], [12], [13]. The energetic method was applied in [14]. A special system of biorthogonal functions was constructed for the solving of the problem for the loaded semi-strip in [15]. The problem for the semi-strip was solved with the help of the stress function in [16], [17]. The Laplaces integral transformation was used for the problems solving in [18]. The approach based on the use of Fadle-Papkovich functions was applied in [19], [20]. The problem of an elastic strip passing between two rollers was considered by means of a numerical method in [21]. The stress fields in a strip containing climb and glide edge dislocations were obtained utilizing a complex Fourier transform in [22]. The dislocation solutions were then employed to perform integral equations for the analysis of a strip weakened by multiple cracks with any configuration and arrangement. In [23] the large deflection evaluating of the thin strip under practical condition of residual stress after cold rolling was investigated using the incompatible von Krmn equations as the governing equations. The problems for the cracked bodies were solved in [24], [25].

Solution of plane problems can be reduced to the solution of singular integral equation (SIE), which often contains fixed singularities. Many scientists investigated fixed singularities and they proposed different approaches to solve SIE with fixed singularities. It is worth stressing some of them: R. V. Duduchava [26], A. P. Soldatov [27], L. G. Mihaylov [28], H. F. Bueekner [29], [30], G. I. Bierman [31], F. Tricomi [32], S. G. Michlin [33], M. G. Kreyn [34], B. Nobl [35], Y. A. Antipov [36], O. V. Onischuk [37] and others.

The solution of a dynamic problem of an elastic strip, coupled to an elastic half-space is reduced to a singular integral equation, which is solved with the help of special quadrature formulae for singular integrals in [38]. The linear integral equation of the third kind with fixed singularities in the kernel was solved by a new generalized version of the collocation method in [39]. The collocation methods were proposed for the numerical solution of the hypersingular integral equation of a notched half-plane problem in [40]. Optimal quadrature formulae for singular integrals with fixed singularity was investigated in [41]. A new generalized spectral relation for the singular operator with two fixed singularities was proposed in [36]. The algebras generated by the Cauchy singular integral operator and integral operators with fixed sin- 
gularities on the unit interval were studied in [42]. A polynomial collocation method for the numerical solution of Cauchy singular integral equations with fixed singularities which are supposed to be of Mellin convolution type was examined in [43]. The quadrature formulae of the highest algebraic accuracy were received for singular integral equations in [44]. It could be applied to the singular integral equations with the generalized Cauchy kernel.

The approach proposed in [45] for the fixed singularities consideration is used in this paper. The special generalized method proposed in [46] was applied to obtain the solution of the SIE with fixed singularities at the end of the integrations interval.

The novelty of the proposed work is not in the consideration of new statements of the problems, but in the new method proposed for the solving of the known problems for a semi-strip. The main advantages of this method are: - the problem is solved without introduction of any new auxiliary function, which is traditional for the solving of the problems for such class. It essentially simplifies the solving process, since the authors can get the calculation formulae for the displacement and stress fields at once; - The fact that this approach allows to reduce this problem to one singular integral equation is as authors think the known advantage of their work. The indication of fixed singularities in the singular kernel of this equation allows calculating as anywhere close to the angular points. The solution was obtained in a quite compact form which provided by the non-standard construction of Green's function as a bilinear expansion. All above allow to propose the indicated methodic for the solving of more complicated mixed elasticity problems for a semi-strip.

\section{The statement of a problem}

The elastic ( $G$ is a share module, $\mu$ is a Poisons coefficient) semi-strip, $0<$ $x<a, 0<y<\infty$ is considered in three cases which defined the boundary conditions on the semi-strip's short edge. At the lateral sides $x=0,0<y<\infty$ and $x=a, 0<y<\infty$ the boundary conditions of fixing are given

$$
u(0, y)=0, v(0, y)=0, u(a, y)=0, v(a, y)=0,0<y<\infty
$$

here $u(x, y)=u_{x}(x, y), v(x, y)=u_{y}(x, y)$ are the displacements which satisfy the Lame's equations. The equations are written in the following form

$$
\left\{\begin{array}{l}
\frac{\partial^{2} u(x, y)}{\partial x^{2}}+\frac{\kappa-1}{\kappa+1} \frac{\partial^{2} u(x, y)}{\partial y^{2}}+\frac{2}{\kappa+1} \frac{\partial^{2} v(x, y)}{\partial x \partial y}=0, \\
\frac{\partial^{2} v(x, y)}{\partial x^{2}}+\frac{\kappa+1}{\kappa-1} \frac{\partial^{2} v(x, y)}{\partial y^{2}}-\frac{2}{\kappa-1} \frac{\partial^{2} u(x, y)}{\partial x \partial y}=0,
\end{array}\right.
$$

where $\kappa=3-4 \mu$ is the Muskchelishvili constant. Three cases of the boundary conditions on the short edge are considered. In the first case (Fig. 1) the semistrip is loaded at the edge $y=0, a_{0}<x<a_{1}$

$$
\sigma_{y}(x, 0)=p(x), \tau_{x y}(x, 0)=0, a_{0}<x<a_{1}
$$




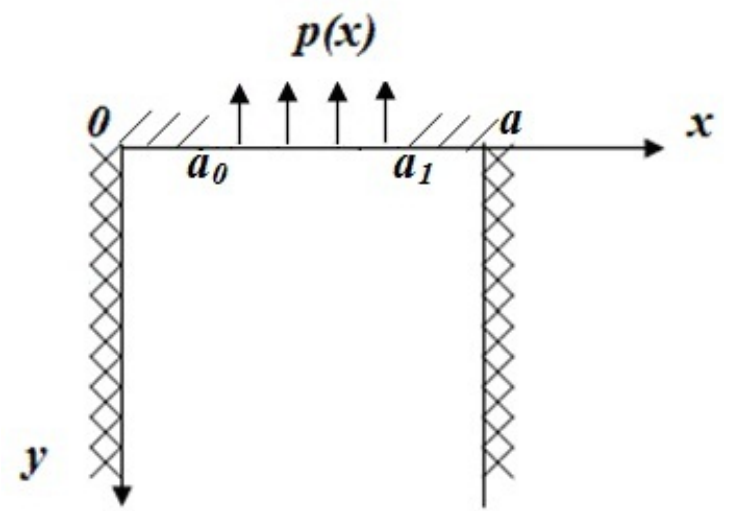

Fig. 1 Geometry and coordinate system of the semi-strip in the first case

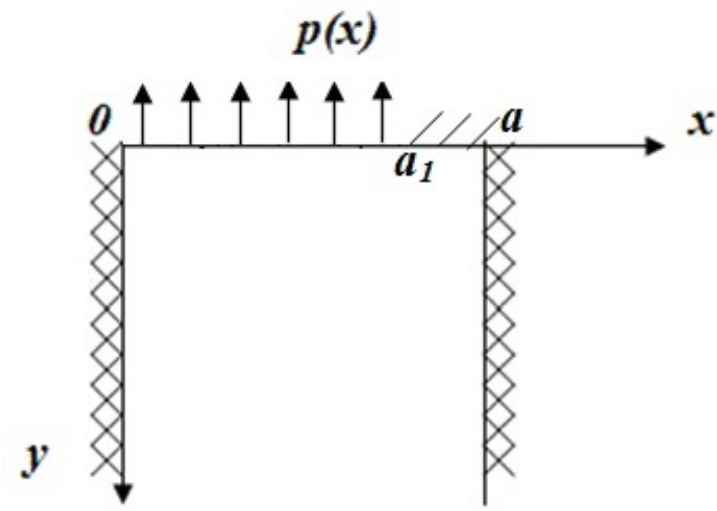

Fig. 2 Geometry and coordinate system of the semi-strip in the second case

where $p(x)$ is the known function. Conditions of the slide contact are executed by the segments $y=0,0<x<a_{0}, a_{1}<x<a$

$$
v(x, 0)=0, \tau_{x y}(x, 0)=0,0<x<a_{0}, a_{1}<x<a
$$

In the second case (Fig. 2) the semi-strip is loaded at the edge $y=0,0<x<$ $a_{1}$

$$
\sigma_{y}(x, 0)=p(x), \tau_{x y}(x, 0)=0,0<x<a_{1}
$$

and conditions of the slide contact are executed by the segments $y=0, a_{1}<$ $x<a$

$$
v(x, 0)=0, \tau_{x y}(x, 0)=0, a_{1}<x<a
$$

In the third case (Fig. 3) the semi-strip is loaded at the edge $y=0,0<x<a$

$$
\sigma_{y}(x, 0)=p(x), \tau_{x y}(x, 0)=0,0<x<a
$$

One needs to solve the boundary value problems (1)-(4), (1)-(2), (5)-(6) and (1)-(2), (7) to estimate the stress state of the semi-strip in three cases. 


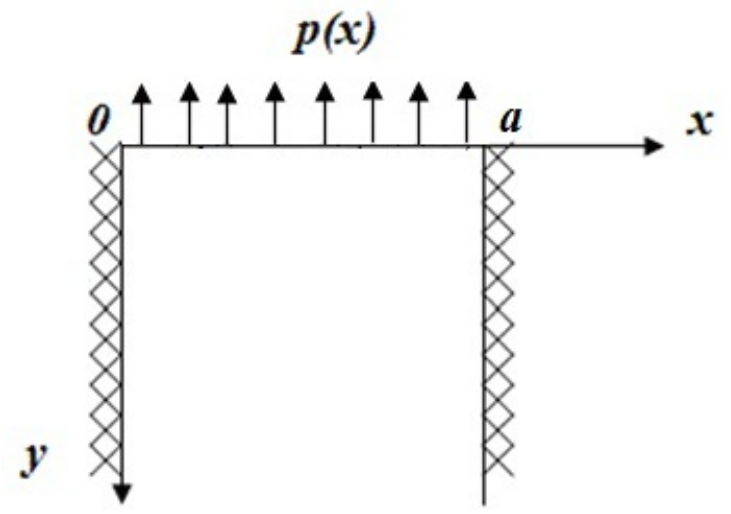

Fig. 3 Geometry and coordinate system of the semi-strip in the third case

\section{The general solution of the problems on the semi-strip stress state estimation}

The initial problem is reduced to the vector boundary problem [47] by the use of the approach [48]. This approach, as it was shown earlier in the monograph [49], allows to present the general solution of the inhomogeneous vector equation as the superposition of the general and partitial solutions of the vector equation. For the construction of the vector equation's particular solution the apparatus based on the construction of the Green's matrix-function [50], [49] was used. The construction of the general solution requires the construction of the matrix system of fundamental solutions obtained earlier [49]. Further it is shown in the article how to make this presentation. More detailed construction of this solution is indicated in the paper [48].

$$
\mathbf{y}_{\beta}(x)=Y_{1}(x)\left(\begin{array}{c}
c_{1} \\
c_{2}
\end{array}\right)+Y_{2}(x)\left(\begin{array}{l}
c_{3} \\
c_{4}
\end{array}\right)+\int_{0}^{a} G(x, \xi) \mathbf{f}(\xi) d \xi
$$

where $Y_{1}(x), Y_{2}(x)$ is the matrix system of the fundamental matrix solutions obtained with the help of the matrix differential calculation apparatus, $c_{i}, i=$ $\overline{1,4}$ are known constants found from boundary conditions $(1), G(x, \xi)$ is the Green's matrix function which was constructed with the help of the matrix integral transformation method as a bilinear expansion, $\mathbf{y}_{\beta}(x)=\left(\begin{array}{l}u_{\beta}(x) \\ v_{\beta}(x)\end{array}\right)$, $\mathbf{f}(x)=\left(\begin{array}{c}\frac{3-\kappa}{\kappa+1} \chi^{\prime}(x) \\ -\beta \frac{\kappa+1}{\kappa-1} \chi(x)\end{array}\right), \beta$ is the Fourier transformation parameter. The new unknown function $\chi(x)=v(x, 0)$ is inputted. The components of the vector $\mathbf{y}_{\beta}(x)$ are components of the Fourier transformations.

The inverse transformations were applied to the components of (8) and substitution of the displacement functions in the boundary conditions (3)-(4), (5)-(6) or (7) leads to the singular integral equations. 
4 Solution of the singular integral equation in the first case

The changing of the variables $\xi=\frac{2 \xi^{*}-\left(a_{0}+a_{1}\right)}{a_{1}-a_{0}}, x=\frac{2 x^{*}-\left(a_{0}+a_{1}\right)}{a_{1}-a_{0}}$ is done for the passing to the integration interval $[-1 ; 1]$. As a result the integral equation is transformed to the form

$$
\frac{d^{2}}{d x^{2}} \int_{-1}^{1} \tilde{\chi}(\xi) \ln \frac{1}{|\xi-x|} d \xi+\int_{-1}^{1} \tilde{\chi}(\xi) \tilde{f}(\xi, x) d \xi=\tilde{r}(x),-1<x<1
$$

here $\tilde{\chi}(\xi)=\chi\left(\frac{\left(a_{1}-a_{0}\right) \xi+\left(a_{0}+a_{1}\right)}{2}\right), \tilde{f}(\xi, x), \tilde{r}(x)$ are known regular functions. The integral equation is solved approximately by the orthogonal polynomials method [45]. This method allows taking into consideration the real singularities of the solution at the ends of the integration interval. With regard of it, the function $\tilde{\chi}(\xi)$ is expanded in the series by the Chebyshev polynomials

$$
\tilde{\chi}(\xi)=\sum_{n=0}^{\infty} s_{n} \sqrt{1-\xi^{2}} U_{n}(\xi), \xi \in[-1 ; 1]
$$

where $U_{n}(x)$ is Chebyshev polynomial of the second type. This expression is substituted into the integral equation (9), and the standard scheme of the orthogonal polynomials method is realized. During it the spectral relationship $[45]$

$\frac{d^{2}}{d x^{2}} \int_{-1}^{1} \ln \frac{1}{|x-y|} \sqrt{1-y^{2}} U_{n}(y) d y=-\pi(n+1) U_{n}(x), n=0,1,2, \ldots,-1<x<1$

is used. As a result, the infinite system of the linear algebraic equations relatively to the unknown coefficients $s_{n}, n=0,1, \ldots$ is obtained

$$
s_{m}+\sum_{n=0}^{\infty} s_{n} d_{m, n}=f_{m}, m=0,1,2, \ldots
$$

here $d_{m, n}=-\frac{1}{\pi(m+1)\left\|U_{m}\right\|^{2}} \int_{-1}^{1} d x \int_{-1}^{1} U_{n}(\xi) U_{m}(x) \sqrt{1-\xi^{2}} \sqrt{1-x^{2}} \tilde{f}(\xi, x) d \xi, f_{m}=$ $-\frac{1}{\pi(m+1)\left\|U_{m}\right\|^{2}} \int_{-1}^{1} \tilde{r}(x) U_{m}(x) \sqrt{1-x^{2}} d x$.

The system (11) is solved by the reduction method [45]. The substitution of the founded constants in the formula (10) and following using of the formulae (8) completes the construction of the problems solution in the first case. 


\section{Solution of the singular integral equation in the second case}

The changing of the variables $\xi=\frac{2 \xi^{*}-a_{1}}{a}{ }_{1}, x=\frac{2 x^{*}-a_{1}}{a}{ }_{1}$ is done for the passing to the integration interval $[-1 ; 1]$. SIE is transformed to the following form

$$
\begin{aligned}
\frac{1}{\pi} \int_{-1}^{1} \frac{\tilde{\chi}(\xi)}{\xi-x} d \xi & +\frac{1}{\pi} \int_{-1}^{1} \tilde{\chi}(\xi)\left[\frac{h_{1}}{\xi+x+2}+\frac{h_{2}(x+1)}{(\xi+x+2)^{2}}+\frac{h_{3}(x+1)(\xi+1)}{(\xi+x+2)^{3}}\right] d \xi+ \\
& +\int_{-1}^{1} \tilde{\chi}(\xi) K(x, \xi) d \xi=\tilde{r}(x),-1<x<1
\end{aligned}
$$

here $\tilde{\chi}(\xi)=\chi^{\prime}\left(\frac{a_{1}(\xi+1)}{2}\right), K(x, \xi), \tilde{r}(x)$ are known regular functions, $h_{1}=-\frac{\kappa^{2}-3}{2 \kappa}, h_{2}=$ $-\frac{2}{\kappa}, h_{3}=\frac{4}{\kappa}$.

The equation (12) is the equation with one fixed singularity. The characteristic equation to (12) was constructed with the help of the asymptotic method [46]. It agrees with the equation in [51] for a problem of a wedge with the angle of openness $\pi / 2$ and Poisson ratio $\mu=0.33$. The roots of the characteristic equation to (12) $\lambda_{k}$ were found.

The generalized method of SIE solving [46], [52] was applied for the solving of the equation (12). According to it the unknown function $\tilde{\chi}(\xi)$ is presented in the following form

$$
\tilde{\chi}(\xi)=\sum_{k=0}^{N-1}\left[s_{k} \rho_{k}^{-}(\xi)+s_{k+N} \rho_{k}^{+}(\xi)\right], \xi \in[-1 ; 1]
$$

where $\begin{gathered}\rho_{2 k}^{-}(\xi)=(1+\xi)^{\Re \lambda_{k}} \cos \left(\Im \lambda_{k} \ln (1+\xi)\right), \\ \rho_{2 k+1}^{-}(\xi)=(1+\xi)^{\Re \lambda_{k}} \sin \left(\Im \lambda_{k} \ln (1+\xi)\right), \rho_{k}^{+}=\frac{T_{n}(\xi)}{\sqrt{1-\xi}}, k=\overline{0, N-1} .\end{gathered}$

The segment $[-1 ; 1]$ is divided on $2 N$ segments by the points $x_{i}: P_{2 N-1}^{\lambda_{0},-0.5}\left(x_{i}\right)=$ $0, i=\overline{0,2 N-1}$. The equation (13) is considered when $x=x_{i}, i=\overline{0,2 N-1}$

$$
\sum_{k=0}^{N-1}\left[s_{k} d_{k i}^{-}+s_{k+N} d_{k i}^{+}\right] d \xi=\tilde{r}\left(x_{i}\right), i=\overline{0,2 N-1}
$$

here $d_{k i}^{ \pm}=\int_{-1}^{1} \rho_{k}^{ \pm}(\xi)\left[\frac{1}{\xi-x_{i}}+\frac{h_{1}}{\xi+x_{i}+2}+\frac{h_{2}\left(x_{i}+1\right)}{\left(\xi+x_{i}+2\right)^{2}}+\frac{h_{3}\left(x_{i}+1\right)(\xi+1)}{\left(\xi+x_{i}+2\right)^{3}}+K\left(x_{i}, \xi\right)\right] d \xi, k=$ $\overline{0, N-1}, i=\overline{0,2 N-1}$.

The expression (14) presents the system of $2 N$ equations with regard to $2 N$ unknown constants $s_{k}$. The substitution of the founded constants in the formula (13) and the following using of the formulae (8) completes the construction of the problems solution in the second case. 


\section{Solution of the singular integral equation in the third case}

The changing of the variables $\xi=\frac{2 \xi^{*}-a}{a}, x=\frac{2 x^{*}-a}{a}$ is done for the passing to the integration interval $[-1 ; 1]$. So SIE is transformed to the form

$$
\begin{gathered}
\frac{1}{\pi} \int_{-1}^{1} \frac{\tilde{\chi}(\xi)}{\xi-x} d \xi+\frac{1}{\pi} \int_{-1}^{1} \tilde{\chi}(\xi)\left[h_{1}\left(\frac{1}{\xi+x-2}+\frac{1}{\xi+x+2}\right)+\right. \\
\left.+h_{2}\left(\frac{x-1}{(\xi+x-2)^{2}}+\frac{x+1}{(\xi+x+2)^{2}}\right)+h_{3}\left(\frac{(x-1)(\xi-1)}{(\xi+x-2)^{3}}+\frac{(x+1)(\xi+1)}{(\xi+x+2)^{3}}\right)\right] d \xi+ \\
+\int_{-1}^{1} \tilde{\chi}(\xi) K(x, \xi) d \xi=\tilde{r}(x),-1<x<1
\end{gathered}
$$

where $\tilde{\chi}(\xi)=\chi^{\prime}\left(\frac{a(\xi+1)}{2}\right), K(x, \xi), \tilde{r}(x)$ are the known regular functions, $h_{1}=$ $-\frac{\kappa^{2}-3}{2 \kappa}, h_{2}=-\frac{2}{\kappa}, h_{3}=\frac{4}{\kappa}$.

The equation (15) is the equation with two fixed singularities. The roots of the equations (15) symbol $\lambda_{k}$ are same to the previous case.

The generalized method of SIE solving [46], [52] was applied for the solving of the equation (15). According to it the unknown function $\tilde{\chi}(\xi)$ is searched in the following form [53]

$$
\tilde{\chi}(\xi)=\sum_{k=0}^{N-1}\left[s_{k} \rho_{k}^{-}(\xi)+s_{k+N} \rho_{k}^{+}(\xi)\right], \xi \in[-1 ; 1]
$$

here $\begin{gathered}\rho_{2 k}^{\mp}(\xi)=(1 \pm \xi)^{\Re \lambda_{k}} \cos \left(\Im \lambda_{k} \ln (1 \pm \xi)\right), \\ \rho_{2 k+1}^{\mp}(\xi)=(1 \pm \xi)^{\Re \lambda_{k}} \sin \left(\Im \lambda_{k} \ln (1 \pm \xi)\right),\end{gathered}, k=\overline{0, N-1}$

The segment $[-1 ; 1]$ is divided on $2 N$ segments by the points $x_{i}: P_{2 N-1}^{\lambda_{0}, \lambda_{0}}\left(x_{i}\right)=$ $0, i=\overline{0,2 N-1}$. The equation (13) is considered when $x=x_{i}, i=\overline{0,2 N-1}$

$$
\sum_{k=0}^{N-1}\left[s_{k} d_{k i}^{-}+s_{k+N} d_{k i}^{+}\right] d \xi=\tilde{r}\left(x_{i}\right), i=\overline{0,2 N-1}
$$

where $d_{k i}^{ \pm}=\int_{-1}^{1} \rho_{k}^{ \pm}(\xi)\left[\frac{1}{\xi-x_{i}}+h_{1}\left(\frac{1}{\xi+x_{i}-2}+\frac{1}{\xi+x_{i}+2}\right)+h_{2}\left(\frac{x_{i}-1}{\left(\xi+x_{i}-2\right)^{2}}+\frac{x_{i}+1}{\left(\xi+x_{i}+2\right)^{2}}\right)\right.$

$$
\left.h_{3}\left(\frac{-1}{\left(x_{i}-1\right)(\xi-1)}+\frac{\left(x_{i}+1\right)(\xi+1)}{\left(\xi+x_{i}-2\right)^{3}}\right)+K\left(x_{i}, \xi\right)\right] d \xi, k=\overline{0, N-1}, i=\overline{0,2 N-1}
$$

The expression (17) presents the system of $2 N$ equations with regard to $2 N$ unknown constants $s_{k}$. The construction of the problems solution in the third case completes by the substitution of the founded constants in the formula (16) and the following using of the formulae (8).

\section{The results of the numerical analyses}

The calculations were done for the elastic semi-strip $\left(g=61.2781955 \cdot 10^{9} \mathrm{~Pa}\right.$, $\mu=0.33), p(x)=1 \mathrm{~Pa}, a=10 \mathrm{~m}$. The purpose of investigation was to find out the limits of the orthogonal polynomials method application. 


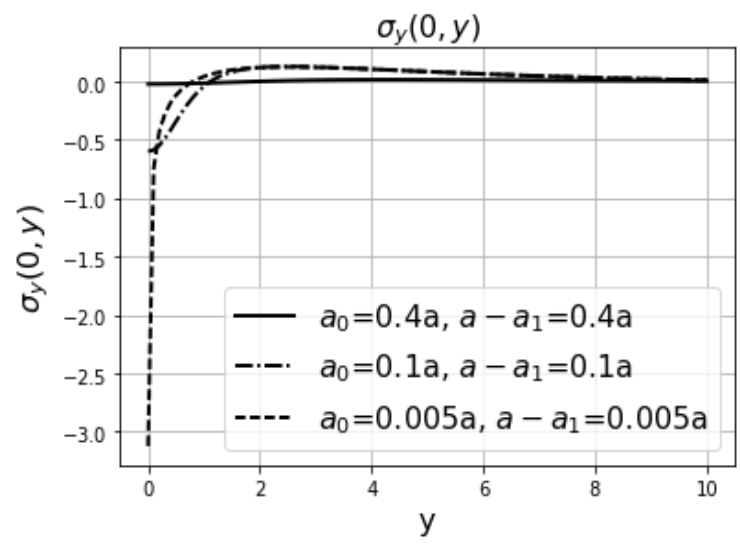

Fig. 4 Normal stresses $\sigma_{y}(0, y), a=10$ in the first case

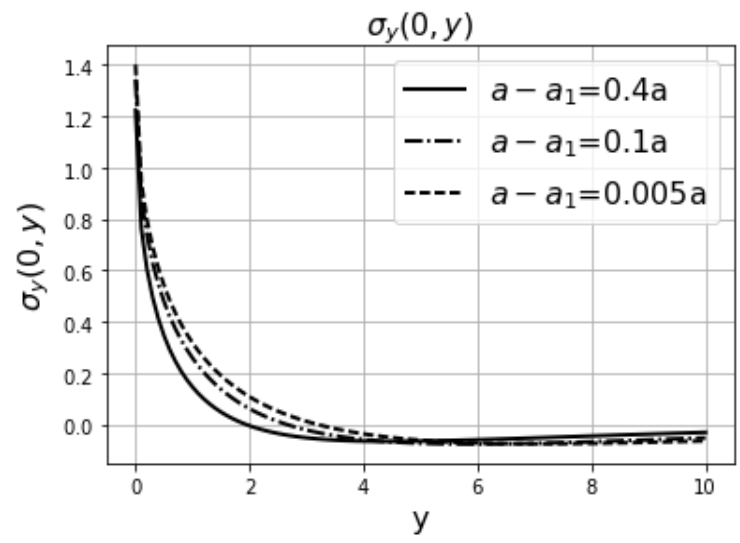

Fig. 5 Normal stresses $\sigma_{y}(0, y), a=10$ in the second case

The Fig. 4 appear the situation when there are no fixed singularities, and the orthogonal polynomials method is applied. At Fig. 4 one can admit that the values of the normal stress $\sigma_{y}$ at the lateral side $x=0$ are increasing when parameters $a_{0}=a-a_{1}$ are decreasing. So the fixed singularity is ought to be considered when $a_{0}=a-a_{1}<0.005 a$.

The situation when the fixed singularity is considered at the left side is presented at Fig. 5, 6. The normal stresses values $\sigma_{y}(0, y)$ at Fig. 5 are almost equal when the parameter $a_{1}$ is changing, which was achieved by the consideration of the fixed singularity at the point $x=0, y=0$. At Fig. 6 one can observe that the values of the normal stress $\sigma_{y}$ at the lateral side $x=a$ are increasing when parameter $a_{1}$ is getting close to the semi-strip's width $a$. So when the load is applied near the right lateral side and $a-a_{1}<0.005 a$ the fixed singularity at the point $x=a, y=0$ should be considered as well as the fixed singularity at the point $x=0, y=0$. 


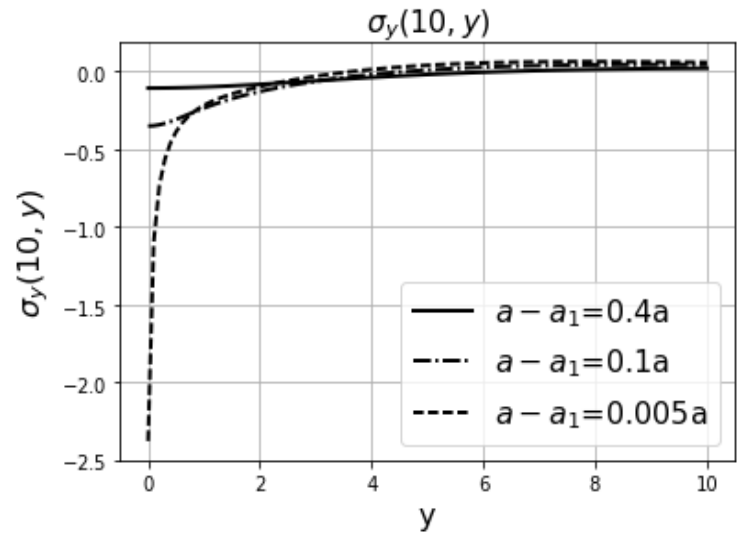

Fig. 6 Normal stresses $\sigma_{y}(10, y), a=10$ in the second case

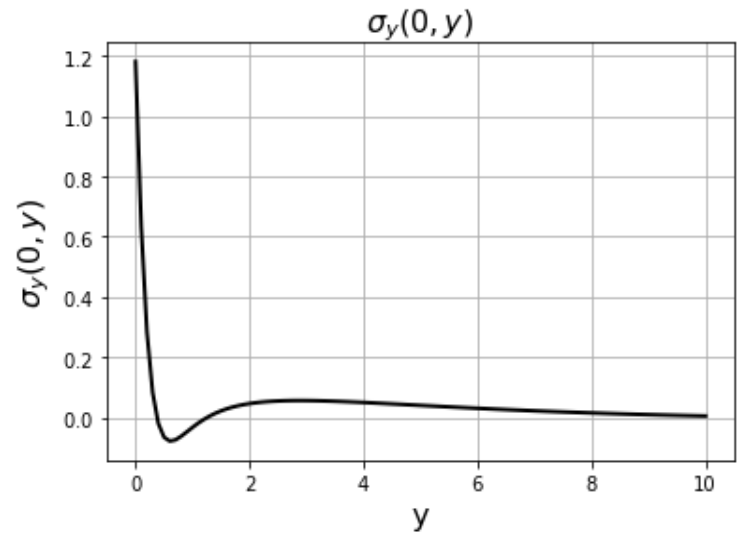

Fig. 7 Normal stresses $\sigma_{y}(0, y), \sigma_{y}(10, y), a=10$ in the third case

The Fig. 7 appears the situation when the fixed singularities are considered at the both sides. The normal stresses values $\sigma_{y}$ changing presented at Fig. 7 at the lateral side $x=0$ are smooth and equal to the normal stresses values $\sigma_{y}$ at the lateral side $x=a$.

\section{The conclusions}

1. The problems for three different types of the load which was given for the shortest edge of the semi-strip were solved. The problem was reduced to the SIE in dependence of the type of the load.

2. The special solving methodic with regard of fixed singularities was proposed in the article. 
3. Zones of the orthogonal polynomials method's applicability are established. When the load is close to the lateral side less than $0.005 a$ fixed singularities should be considered. Thus the numerical calculations show that without consideration of the fixed singularity the use of classical-type singularities allows to get to the angular point in average to the distance no more than $0.005 a$ (Fig. 4), while its consideration allows to get to this point anywhere close (Fig. 7).

Acknowledgements Gratitude for Simon Dyke for the editing of the articles text.

\section{References}

1. Babeshko VA, Babeshko OM, Evdokimova OV. On the method of block element. Mechanics of Solids 2010;45:437-444.

2. Bogy DB. Solution of the plane end problem for a semi-infinite elastic strip. J Appl Math Phys 1975;26:749-769.

3. Ling CB, Cheng FH. Stresses in a semi-infinite strip. Int J Eng Sci 1967;5(2):155.

4. Pickett G, Jyengar KTS. Stress concentrations in post-tensioned prestressed concrete beams. J Technol India 1956;1(2).

5. Jamasida. Research of the tensions in semi-infinite strip under acting forces applied to its edge. Trans Japan Soc Mech Engrs 1954;20(95):466.

6. Vorovich II, Kopasenko VV. Some problems of elasticity theory for the semi-strip (in Russian). Prikladnaya matematica i mekchanica 1966;30(1):128-136.

7. Koiter W, Alblas J. On the bending of cantilever rectangular Plates. Proc Koninke Nederl Acad wet B 1954;57(2).

8. Aglovyan LA, Gevorkyan RS. About some mixed problems of elasticity theory for the semi-strip (in Russian). News of academy of science Armenian SSR, Mechanics 1970;23(3):3-13.

9. Zorski H. A semi-infinite strip with discontinuous boundary conditions. Arch Mech Stosowanej 1958;10(3):371-397.

10. Zamyatin VM, Makhov AV, Svetashkov AA. Solving of plane problems of elasticity for a strip with the help of diagonalized system of equilibrium equations (in Russian). Izvestiya Tomskogo politehnicheskogo universiteta 2006;309(6):135-139.

11. Trapeznikov LP. Influence lines for the normal tensions in semi-strip (in Russian). News of USSR n-i of the hydromechanical institute 1963;73.

12. Kolchin GB, Plyat ShN, Sheynker NYa. Some thermoelastic problems for the rectangular regions (in Russian). Shtiica, 1980.

13. Suchevan VG. The tensioned state of the elastic semi-strip with fixed edges (in Russian). Matematiceskie issledovaniya 1976;40:122-135.

14. Thecaris P. The stress distribution in a semi-infinite strip subjected to a concentrated load. Trans J Appl Mech 1959;26(3):401406.

15. Johnson MW, Little RW. The semi-infinite elastic strip. Q Appl Math 1965;22(4):335344 .

16. Horvay G. The end problem of rectangular strips. J Appl Mech 1953;20:87-94.

17. Horvay G, Born J. Some mixed boundary-value problems of the semi-infinite strip. Journal of Applied Mechanics 1957;24(2):261-268.

18. Benthem JP. A Laplace transform method for the solution of semi-infinite and finite strip problems in stress analysis. Quart J Mech and Appl Math 1963;16(4):413-429.

19. Gogoleva OS. The examples of solutions of the first main boundary problem of elasticity theory in the semi-strip (symmetrical problem) (in Russian). Journal Omskiy gosudarstvenniy universitet 2012;145(9):138-142.

20. Kovalenko MD, Menshova I, Shulyakovskaya TD. Expansions in Fadle-Papkovich functions: Examples of solutions in a half-strip. Mechanics of Solids 2013;48:584-602, DOI 10.3103/S0025654413050154. 
21. Bentall RH, Johnson KL. An Elastic Strip in Plane Rolling Contact. International Journal of Mechanical Science 1968;10(8):637-663.

22. Fotuhi AR, Fariborz SJ. Stress analysis in a cracked strip. International Journal of Mechanical Sciences 2008;50(2):132-142.

23. Nong Li, Hongbo Li, Jie Zhang, Ning Kong, Shenghui Jia. Composite expansion-Ritz method to post buckling problem of thin cold rolled strip. International Journal of Mechanical Sciences 2017; 128?129:379-388.

24. Guz AN, Guz IA, Men?shikov AV, Men?shikov VA. Stress-intensity factors for materials with interface cracks under harmonic loading. Int Appl Mech 2011;46:1093. https://doi.org/10.1007/s10778-011-0401-1.

25. Mykhas'kiv V, Stankevych V, Zhbadynskyi I, Zhang Ch. 3-D dynamic interaction between a penny-shaped crack and a thin interlayer joining two elastic half-spaces. International Journal of Fracture 2009;159(2):137-149.

26. Duduchava RV. Integral equations with fixed singularities. BG Teubner, 1979

27. Soldatov AP. A problem in function theory. Diff Equations 1973;9(2):248253.

28. Mihailov LG. Integral equations with the kernel which is homogonous of the degree -1 (in Russian). Dopish, Dushanbe, 1966.

29. Bueekner HF. Some stress singularities and their computation by means of integral equations. Boundary problems in differential equation. Univ Wisconsin Press, Madison, 1960:215-230.

30. Bueekner HF. On a class of singular integral equations. J Mathem Anal and Appl $1966 ; 14: 392-426$

31. Bierman GI. A particular class of singular integral equations. J Appl Mathem 1971;20(1):99-109.

32. Tricomi F. Atti Accad Naz Lincei. 1932;5(14):134247.

33. Michlin SG, Prossdorf S. Singular Integral Operators. Akademie Verlag, Berlin, 1986.

34. Kreyn MG. Integral equation on the semi-line with the kernel, which depend on arguments subtraction (in Russian). UMN 1958;13(5):3-120.

35. Noble B. Methods Based on the Wiener-Hopf Technique For the Solution of Partial Differential Equations. Pergamon Press, Belfast, Northern Ireland, 1958.

36. Antipov YA. Singular integral equations with two fixed singularities and applications to fractured composites. The Quarterly Journal of Mechanics and Applied Mathematics $2015 ; 68(4)$.

37. Onischuk OV, Popov GYa, Farshayt PG. The problem about bend of rectangular plate with linear pile, which goes on the fixed side by one end. Mechanics of solids 1988;6:160167 .

38. Popov VG. A dynamic contact problem which reduces to a singular integral equation with two fixed singularities. Journal of Applied Mathematics and Mechanics 2012;76(3):348-357.

39. Gabbasov NS. Methods for solving an integral equation of the third kind with fixed singularities in the kernel. Differential Equations 2009;45(9):1341-1348.

40. Capobianco MR, Criscuolo G, Junghanns P. On the numerical solution of a hypersingular integral equation with fixed singularities. Operator Theory: Advances and Applications 187:95-116.

41. Shabozov MSh. An approach to the investigation of optimal quadrature formulas for singular integrals with fixed singularity. Ukrainian Mathematical Journal 1995;47(9):14791485 .

42. Duduchava R, Krupnik N, Shargorodsky E. An algebra of integral operatirs with fixed singularities in kernels. Integral Equations and Operator Theory 1999;33(4):406-425

43. Junghanns P, Rathsfeld. A On polynomial collocation for Cauchy singular integral equations with fixed singularities. Integral Equations and Operator Theory 2002;43(2):155-176.

44. Sahakyan AV. Method of discrete singularities for solution of singular integral and integro-differential equations. Proceedings of A Razmadze Mathematical Institute 2011:156.

45. Popov GYa. The elastic stress' concentration around dies, cuts, thin inclusions and reinforcements (in Russian). Nauka, Moskow, 1982.

46. Kryvyi OF. Mutual influence of an interface tunnel crack and an interface tunnel inclusion in a piecewise homogeneous anisotropic space. Journ of Mathematical Sciences 2015;208(4):409-416. 
47. Vaysfeld ND, Zhuravlova ZYu. Two-dimensional mixed problem of thermoelasticity for a semistrip. Journal of Mathematical Sciences 2018;228(2):105-121. DOI 10.1007/s10958017-3609-8

48. Vaysfeld ND, Zhuravlova ZYu. On one new approach to the solving of an elasticity mixed problem for the semi-strip. Acta Mechanica 2015;226(12):4159-4172. DOI: 10.1007/s00707015-1452-x.

49. Popov GYa, Abdimanov SA, Ephimov VV. Green?s functions and matrixes of the onedimensional boundary problems (in Russian). Raczah, Almati, 1999.

50. Naymark MA. Linear differational operators (in Russian). Nauka, Moskow, 1969.

51. Uflyand YaS. Integral transformations in the problems of the elasticity theory (in Russian). Nauka, Leningrad, 1967.

52. Vaysfeld N, Kryvyi O, Zhuravlova Z. On the stress investigation at the edge of the fixed elastic semi-strip. Frattura ed Integrita Strutturale 2016;38:1-11. DOI: 10.3221/IGFESIS.38.01.

53. Zhuravlova Z. Stress analysis near the tips of a transverse crack in an elastic semi-strip. Appl Math Mech Engl Ed 2017. DOI 10.1007/s10483-017-2217-6. 